\title{
Errors in Absolute Orientation (II)
}

\section{by Kazuo Muraoka}

\section{Stereo-comparator $の$ 場合}

前報 $[2,4]$ に抢けると同様にStereo-comparator に よって写真の測定を行ない, 独立な測定要素が第 1 写 真の写真座標 $\left(x_{1 n}, y_{1 n}\right)$ 之視差 $x_{2 n}-x_{1 n}=q_{n}, y_{2 n}-$ $y_{1 n}=p_{n}$ の場合を想定する。乙の場合独立な誤差要素 $\delta x_{1 n}, \delta y_{1 n}, \delta q_{n}, \delta p_{n}$ の関数として諸誤差を整理する。 Table III についててれを行えば， $\delta p_{n}, \delta q_{n}$ について は同表において，それぞれ $\delta y_{2 n}, \delta x_{2 n}$ を $\delta p_{n}, \delta q_{n}$ と 読み換えたすのに全く同じになるが， $\delta y_{1 n}, \delta x_{1 n}$ につ いては Table VIに示されるようになる。従ってbに 前項と同じ数值を与えた場合, Table IV に相当する
表のうち $\delta y_{1 n}, \delta x_{1 n}$ に関する部分は Table VII こな る。測定值が絶対值一様な誤差を持つとして誤差伝播 の法則を用いて Table V の第 2 列に相当する值を Table VIII の第 2 列の如くにえる。さらに

$$
\delta p=\delta y=\delta q=\delta x=\varepsilon^{\prime} \mu
$$

とすれば，同表の第 3 列の如き值となる。基線長 800 $\mathrm{m}, \varepsilon^{\prime}=10 \mu$ とすれば同表右端列をうる。

\section{IV. 与点誤差について}

\section{1. 与点誤差の影響}

与点となる測地座標に誤差があるときには，それが

Table VI 座標誤差 Displacement of co-ordinates***

(a) $\delta X$

\begin{tabular}{|c|c|c|c||c|c|c|c|}
\hline$\downarrow$ & $\delta y_{11}$ & $\delta y_{12}$ & $\delta y_{13}$ & $\delta x_{11}$ & $\delta x_{12}$ & $\delta x_{13}$ & \\
\hline 1 & 0 & $-\frac{1}{11 b}$ & $-\frac{2}{11 b}$ & $+\frac{20}{33 b}$ & $-\frac{7}{33 b}$ & $-\frac{1}{33 b}$ & 6 \\
2 & $+\frac{1}{11 b}$ & 0 & $-\frac{1}{11 b}$ & $-\frac{7}{33 b}$ & $+\frac{26}{33 b}$ & $-\frac{7}{33 b}$ & 5 \\
3 & $+\frac{2}{11 b}$ & $+\frac{1}{11 b}$ & 0 & $-\frac{1}{33 b}$ & $-\frac{7}{33 b}$ & $+\frac{20}{33 b}$ & 4 \\
4 & $-\frac{2}{11 b}$ & $-\frac{1}{11 b}$ & 0 & $-\frac{10}{33 b}$ & $-\frac{4}{33 b}$ & $+\frac{2}{33 b}$ & 3 \\
5 & $-\frac{2}{11 b}$ & 0 & $+\frac{1}{11 b}$ & $-\frac{4}{33 b}$ & $-\frac{4}{33 b}$ & $-\frac{4}{33 b}$ & 2 \\
6 & 0 & $+\frac{1}{11 b}$ & $+\frac{2}{11 b}$ & $+\frac{2}{33 b}$ & $-\frac{4}{33 b}$ & $-\frac{10}{33 b}$ & 1 \\
\hline & $\delta y_{16}$ & $\delta y_{15}$ & $\delta y_{14}$ & $\delta x_{16}$ & $\delta x_{15}$ & $\delta x_{14}$ & $\leftarrow$ \\
\hline
\end{tabular}

(b) $\delta Y$

\begin{tabular}{|c|c|c|c||c|c|c|c|}
\hline$\downarrow$ & $\delta y_{11}$ & $\delta y_{12}$ & $\delta y_{13}$ & $\delta x_{11}$ & $\delta x_{12}$ & $\delta x_{13}$ & \\
\hline 1 & $-\frac{20}{33 b}$ & $+\frac{7}{33 b}$ & $+\frac{1}{33 b}$ & 0 & $-\frac{1}{11 b}$ & $-\frac{2}{11 b}$ & 6 \\
2 & $+\frac{7}{33 b}$ & $-\frac{26}{33 b}$ & $+\frac{7}{33 b}$ & $+\frac{1}{11 b}$ & 0 & $-\frac{1}{11 b}$ & 5 \\
3 & $+\frac{1}{33 b}$ & $+\frac{7}{33 b}$ & $-\frac{20}{33 b}$ & $+\frac{2}{11 b}$ & $+\frac{1}{11 b}$ & 0 & 4 \\
4 & $+\frac{10}{33 b}$ & $+\frac{4}{33 b}$ & $-\frac{2}{33 b}$ & $-\frac{2}{11 b}$ & $-\frac{1}{11 b}$ & 0 & 3 \\
5 & $+\frac{4}{33 b}$ & $+\frac{4}{33 b}$ & $+\frac{4}{33 b}$ & $-\frac{1}{11 b}$ & 0 & $+\frac{1}{11 b}$ & 2 \\
6 & $-\frac{2}{33 b}$ & $+\frac{4}{33 b}$ & $+\frac{10}{33 b}$ & 0 & $+\frac{1}{11 b}$ & $+\frac{2}{11 b}$ & 1 \\
\hline & $\delta y_{16}$ & $\delta y_{15}$ & $\delta y_{14}$ & $\delta x_{16}$ & $\delta x_{15}$ & $\delta x_{14}$ & $\leftarrow$ \\
\hline
\end{tabular}

* The contributions from $\delta p_{n}$ and $\delta q_{n}$ are the same as those from $\delta y_{2 n}$ and $\delta x_{2 n}$ respectively in Table III. 
Table VII 座標誤差 Displcement of co-ordinates

(a) $\delta X \times 10^{6}$

\begin{tabular}{|c|c|c|c||c|c|c|c|}
\hline$\downarrow$ & $\delta y_{11}$ & $\delta y_{12}$ & $\delta y_{13}$ & $\delta x_{11}$ & $\delta x_{12}$ & $\delta x_{13}$ & \\
\hline 1 & 0 & -1.1 & -2.3 & +7.6 & -2.7 & -0.4 & 6 \\
2 & +1.1 & 0 & -1.1 & -2.7 & +9.8 & -2.7 & 5 \\
3 & +2.3 & +1.1 & 0 & -0.4 & -2.7 & +7.6 & 4 \\
4 & -2.3 & -1.1 & 0 & -3.8 & -1.5 & +0.8 & 3 \\
5 & -1.1 & 0 & +1.1 & -1.5 & -1.5 & -1.5 & 2 \\
6 & 0 & +1.1 & +2.3 & +0.8 & -1.5 & -3.8 & 1 \\
\hline & $\delta y_{16}$ & $\delta y_{15}$ & $\delta y_{14}$ & $\delta x_{16}$ & $\delta x_{15}$ & $\delta x_{14}$ & $\leftarrow \uparrow$ \\
\hline
\end{tabular}

(b) $\delta Y \times 10^{6}$

\begin{tabular}{|c|c|c|c||c|c|c|c|}
\hline$\downarrow$ & $\delta y_{11}$ & $\delta y_{12}$ & $\delta y_{13}$ & $\delta x_{11}$ & $\delta x_{12}$ & $\delta x_{13}$ & \\
\hline 1 & -7.6 & +2.7 & +0.4 & 0 & -1.1 & -2.3 & 6 \\
2 & +2.7 & -9.8 & +2.7 & +1.1 & 0 & -1.1 & 5 \\
3 & +0.4 & +2.7 & +7.6 & +2.3 & +1.1 & 0 & 4 \\
4 & +3.8 & +1.5 & -0.8 & -2.3 & -1.1 & 0 & 3 \\
5 & +1.5 & +1.5 & +1.5 & -1.1 & 0 & +1.1 & 2 \\
6 & -0.8 & +1.5 & +3.8 & 0 & +1.1 & +2.3 & 1 \\
\hline & $\delta y_{16}$ & $\delta y_{15}$ & $\delta y_{14}$ & $\delta x_{16}$ & $\delta x_{15}$ & $\delta x_{14}$ & $\leftarrow \uparrow$ \\
\hline
\end{tabular}

Table VIII 統計的座標誤差 Statistical displacement of co-ordinates

\begin{tabular}{|c|c|c|c|}
\hline & $\delta p_{n}=\delta p, \quad \delta y_{1 n}=\delta y, \quad \delta q_{n}=\delta q . \quad \delta x_{1 n}=\delta x$ & $\delta \not p=\delta y=\delta q=\delta x=\varepsilon^{\prime} \mu$ & $\begin{aligned} \text { Base } & =800 \mathrm{~m} \\
\varepsilon^{\prime} & =10 \mu\end{aligned}$ \\
\hline$\delta X_{1}, 3$ & $\pm \sqrt{(4.55)^{2}(\delta p)^{2}+(3.61)^{2}(\delta y)^{2}+(3.23)^{2}(\delta q)^{2}+(9.08)^{2}(\delta x)^{2}}$ & $\pm 11.3 \times 10^{-6} \varepsilon^{\prime}$ & $\pm 9.0 \mathrm{~cm}$ \\
\hline$\delta X_{2}$ & $\pm \sqrt{(1.56)^{2}(\delta p)^{2}+(2.20)^{2}(\delta y)^{2}+(2.23)^{2}(\delta q)^{2}+(10.83)^{2}(\delta x)^{2}}$ & \pm 11.4 & \pm 9.1 \\
\hline$\delta X_{4}, 6$ & $\pm \sqrt{(7.02)^{2}(\delta p)^{2}+(3.61)^{2}(\delta y)^{2}+(8.81)^{2}(\delta q)^{2}+(9.08)^{2}(\delta x)^{2}}$ & \pm 14.9 & \pm 11.9 \\
\hline$\delta X_{5}$ & $\pm \sqrt{(1.56)^{2}(\delta \not p)^{2}+(2.20)^{2}(\delta y)^{2}+(11.28)^{2}(\delta q)^{2}+(10.83)^{2}(\delta x)^{2}}$ & \pm 15.9 & \pm 12.8 \\
\hline$\delta Y_{1}, 3$ & $\pm \sqrt{(8.76)^{2}(\delta b)^{2}+(9.09)^{2}(\delta y)^{2}+(9.85)^{2}(\delta q)^{2}+(3.61)^{2}(\delta x)^{2}}$ & \pm 14.7 & \pm 11.7 \\
\hline$\delta Y_{2}$ & $\pm \sqrt{(10.47)^{2}(\delta p)^{2} \pm(10.79)^{2}(\delta y)^{2}+(5.40)^{2}(\delta q)^{2}+(2.20)^{2}(\delta x)^{2}}$ & \pm 16.1 & \pm 12.9 \\
\hline$\delta Y_{4}, 6$ & $\pm \sqrt{(6.93)^{2}(\delta b)^{2}+(9.09)^{2}(\delta y)^{2}+(8.81)^{2}(\delta q)^{2}+(3.61)^{2}(\delta x)^{2}}$ & \pm 14.9 & \pm 11.9 \\
\hline$\delta Y_{5}$ & $\pm \sqrt{(6.65)^{2}(\grave{\delta} b)^{2}+(10.79)^{2}(\delta y)^{2}+(3.30)^{2}(\delta q)^{2}+(2.20)^{2}(\delta x)^{2}}$ & \pm 13.2 & \pm 10.5 \\
\hline$\delta Z_{1}, 3,4,6$ & 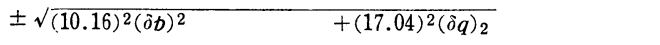 & \pm 19.8 & \pm 15.8 \\
\hline$\delta Z_{2}, 5$ & $\pm 19.11|\delta q q|$ & \pm 19.1 & \pm 15.3 \\
\hline
\end{tabular}

また絶対標定に影響をおよぼす。測地座標に $\Delta X, \Delta Y$, $\Delta Z$ なる誤差があったとすれば，(9) 式において $X, Y$, $Z$ の代りに $X+\Delta X, Y+\Delta Y, Z+\Delta Z$ と置いて計算をす すめるととになる。既述のように, $(\Delta X)^{2},(\Delta Y)^{2}$, $(\Delta Z)^{2},(\delta x)(\Delta X),(\delta x)(\Delta Y) \cdots \cdots$, およびより高次項 を省略するととにより，与点誤差による標定要素への 䚻寄を $X_{0}^{\prime}, Y_{0}^{\prime}, Z_{0}{ }^{\prime}, \delta k^{\prime}, \delta K^{\prime}, \delta \Phi^{\prime}, \delta \Omega^{\prime}$, 標定後の座標誤 差を $\delta X^{\prime}, \delta Y^{\prime}, \delta Z^{\prime}$ とすれば標定要素は

$$
\begin{aligned}
& X_{0}+X_{0}^{\prime}, Y_{0}+Y_{0}^{\prime}, Z_{0}+Z_{0}^{\prime}, 1+\delta k+\delta k^{\prime}, \\
& \delta K+\delta K^{\prime}, \delta \Phi+\delta \Phi^{\prime}, \delta \Omega+\delta \Omega^{\prime}
\end{aligned}
$$

で表わされ, 標定後の座標誤差は

$$
\delta X+\delta X^{\prime}, \delta Y+\delta Y^{\prime}, \delta Z+\delta Z^{\prime}
$$

であり, 基準点からのずれは

$$
\begin{aligned}
& \delta X+\delta X^{\prime}-\Delta X, \quad \delta Y+\delta Y^{\prime}-\Delta Y, \\
& \delta Z+\delta Z^{\prime}-\Delta Z
\end{aligned}
$$

である。

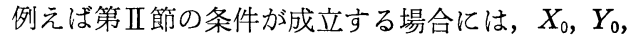
$Z_{0}, 1+\delta k, \delta K, \delta \Phi, \delta \Omega$ は (16) 式によって与えられ, 与点誤差による要素はそれぞれ

$$
\begin{aligned}
& X_{0}^{\prime}=+\frac{1}{n} \Sigma \Delta X, Y_{0}^{\prime}=+\frac{1}{n} \Sigma \Delta Y, \\
& Z_{0}^{\prime}=+\frac{1}{n} \Sigma \Delta Z \\
& \delta k^{\prime}=\frac{\Sigma(X \Delta X+Y \Delta Y+Z \Delta Z)}{\Sigma\left(X^{2}+Y^{2}+Z^{2}\right)}, \\
& \delta K^{\prime}=-\frac{\Sigma(X \Delta Y-Y \Delta X)}{\Sigma\left(X^{2}+Y^{2}\right)}, \\
& \delta \Phi^{\prime}=-\frac{\Sigma(X \Delta Z-Z \Delta X)}{\Sigma\left(Z^{2}+X^{\prime 2}\right)}, \\
& \delta \Omega^{\prime}=-\frac{\Sigma(Z \Delta Y-Y \Delta Z)}{\Sigma\left(Y^{2}+Z^{2}\right)}
\end{aligned}
$$

となる。標定後の座標は(10)式に(22)を代入してえら れる。 


\section{6 点による場合}

前節の例の場合において与点の測地座標が誤差を持 ち, Table I の中の測地座標が Table IX のように 表わされている場合にこの誤差が標定におよぼす影響 を考察してみよう。

Table IX の值および(19)を(10)式に代入して, 標 定後の座標を

$$
X+\delta X+\delta X^{\prime}, Y+\delta Y+\delta Y^{\prime}, Z+\delta Z+\delta Z^{\prime}
$$

とうる。ただし, $\delta X, \delta Y, \delta Z$ は (17) 式で与えられ, $\delta X^{\prime}, \delta Y^{\prime}, \delta Z^{\prime}$ は次式で与えられる。

$$
\begin{aligned}
& \delta X_{1}^{\prime}=X_{0}^{\prime}-\frac{1}{2} \delta k^{\prime}-\delta K^{\prime}, \quad \delta Y_{1}^{\prime}=Y_{0}^{\prime}-\delta k^{\prime}+\frac{1}{2} \delta K^{\prime}, \quad \delta Z_{1}^{\prime}=Z_{0}^{\prime}+\frac{1}{2} \delta \Phi^{\prime}-\delta \Omega^{\prime} \\
& \delta X_{2}^{\prime}=X_{0}^{\prime}-\frac{1}{2} \delta k^{\prime}, \quad \delta Y_{2}^{\prime}=Y_{o}^{\prime} \quad+\frac{1}{2} \delta K^{\prime}, \quad \delta Z_{2}^{\prime}=Z_{0}^{\prime}+\frac{1}{2} \delta \Phi^{\prime} \\
& \delta X_{3}{ }^{\prime}=X_{0}-\frac{1}{2} \delta k^{\prime}+\delta K^{\prime}, \quad \delta Y_{3}{ }^{\prime}=Y_{0}{ }^{\prime}+\delta k^{\prime}+\frac{1}{2} \delta K^{\prime}, \quad \delta Z_{3}{ }^{\prime}=Z_{0}{ }^{\prime}+\frac{1}{2} \delta \Phi^{\prime}+\delta \Omega^{\prime} \\
& \delta X_{4}{ }^{\prime}=X_{0}{ }^{\prime}+\frac{1}{2} \delta k^{\prime}-\delta K^{\prime}, \quad \delta Y_{4}^{\prime}=Y_{0}^{\prime}-\delta k^{\prime}-\frac{1}{2} \delta K^{\prime}, \quad \delta Z_{4}^{\prime}=Z_{0}^{\prime}-\frac{1}{2} \delta \Phi^{\prime}-\delta \Omega^{\prime} \\
& \delta X_{5}^{\prime}=X_{0}^{\prime}+\frac{1}{2} \delta k^{\prime}, \quad \delta Y_{5}^{\prime}=Y_{0}^{\prime} \quad-\frac{1}{2} \delta K^{\prime}, \quad \delta Z_{5}^{\prime}=Z_{0}^{\prime}-\frac{1}{2} \delta \Phi^{\prime} \\
& \delta X_{6}{ }^{\prime}=X_{0}{ }^{\prime}+\frac{1}{2} \delta k^{\prime}+\delta K^{\prime}, \quad \delta Y_{6}{ }^{\prime}=Y_{0}^{\prime}+\delta k^{\prime}-\frac{1}{2} \delta K^{\prime}, \quad \delta Z_{6}^{\prime}=Z_{0}{ }^{\prime}-\frac{1}{2} \delta \Phi^{\prime}+\delta \Omega^{\prime}
\end{aligned}
$$

6 点を全部用いて標定した場合には(22)式は

$$
\begin{aligned}
& X_{0}^{\prime}=\frac{1}{6}\left(\Delta X_{1}+\Delta X_{2}+\Delta X_{3}+\Delta X_{4}+\Delta X_{5}+\Delta X_{6}\right) \\
& Y_{0}^{\prime}=\frac{1}{6}\left(\Delta Y_{1}+\Delta Y_{2}+\Delta Y_{3}+\Delta Y_{4}+\Delta Y_{5}+\Delta Y_{6}\right) \\
& Z_{0}^{\prime}=\frac{1}{6}\left(\Delta Z_{1}+\Delta Z_{2}+\Delta Z_{3}+\Delta Z_{4}+\Delta Z_{5}+\Delta Z_{6}\right) \\
& \delta k^{\prime}=-\frac{1}{11}\left(\Delta X_{1}+\Delta X_{2}+\Delta X_{3}-\Delta X_{4}-\Delta X_{5}-\Delta X_{6}+2 \Delta Y_{1}-2 \Delta Y_{3}+2 \Delta Y_{4}-2 \Delta Y_{6}\right) \\
& \delta K^{\prime}=-\frac{1}{11}\left(2 \Delta X_{1}-2 \Delta X_{3}+2 \Delta X_{4}-2 \Delta X_{6}-\Delta Y_{1}-\Delta Y_{2}-\Delta Y_{3}+\Delta Y_{4}+\Delta Y_{5}+\Delta Y_{6}\right) \\
& \delta \Phi^{\prime}=-\frac{1}{3}\left(-\Delta Z_{1}-\Delta Z_{2}-\Delta Z_{3}+\Delta Z_{4}+\Delta Z_{5}+\Delta Z z_{6}\right) \\
& \delta \Omega^{\prime}=-\frac{1}{4}\left(\Delta Z_{1}-\Delta Z_{3}+\Delta Z_{4}-\Delta Z_{6}\right)
\end{aligned}
$$

となり，乙れを(24)式に代入するととによって $\delta X^{\prime}$, $\delta Y^{\prime}, \delta Z^{\prime}$ をえる。(Table X)

$\Delta X, \Delta Y, \Delta Z$ がすべて互いに独立であるとして, 誤 差伝摇の法則を用いると,

$$
|\Delta X|=|\Delta Y|=\nu,|\Delta Z|=\rho
$$

なる場合には, Table X より

$$
\begin{aligned}
& \delta X_{1,3,4,6}^{\prime}, \delta Y_{1,{ }_{3}, 4,6}= \pm 0.54 \text {, , } \\
& \delta Z^{\prime}{ }_{1,3,4,6}= \pm 0.72 \rho \\
& \delta X^{\prime}{ }_{2,5}, \delta Y^{\prime}{ }_{2,5}= \pm 0.46 \nu \text {, } \\
& \delta Z^{\prime}{ }_{2,5}= \pm 0.57 \rho
\end{aligned}
$$

をうる。

基準点の閉塞への帰寄は(21)を用いて

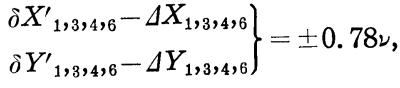

$$
\begin{aligned}
& \delta Z_{1,3,4,6}^{\prime}-\Delta Z_{1,3,4,6}= \pm 0.65 \rho \text {, } \\
& \left.\begin{array}{l}
\delta X^{\prime}{ }_{2,5}-\Delta X_{2,5} \\
\delta Y^{\prime}{ }_{2,5}-\Delta Y_{2,5}
\end{array}\right\}= \pm 0.89 \nu, \\
& \delta Z^{\prime}{ }_{2,5}-\Delta Z_{2},{ }_{5}= \pm 0.82 \rho
\end{aligned}
$$

である。

(27)式と(28)式を比較すると, 測地座標の真值から 
Table X ( I ) $\delta X^{\prime}$

\begin{tabular}{|c|c|c|c|c|c|c|c|c|c|c|c|c|c|}
\hline & $\Delta X_{1}$ & $\Delta X_{2}$ & $\Delta X_{3}$ & $\Delta X_{4}$ & $\Delta X_{5}$ & $\Delta X_{6}$ & $\Delta Y_{1}$ & $\Delta Y_{2}$ & $\Delta Y_{3}$ & $\Delta Y_{4}$ & $\Delta Y_{5}$ & $\Delta Y_{6}$ & $\Delta Z_{1} \sim \Delta Z_{6}$ \\
\hline 1 & +-13 & $\begin{array}{c}7 \\
+\quad 33\end{array}$ & $+\frac{1}{33}$ & $+\begin{array}{r}10 \\
+33\end{array}$ & $+\frac{4}{33}$ & $-\frac{2}{33}$ & 0 & $-\begin{array}{c}1 \\
11\end{array}$ & $-\frac{2}{11}$ & $+\frac{2}{11}$ & $+-\frac{1}{11}-$ & 0 & 0 \\
\hline 2 & $+\begin{array}{r}7 \\
+33\end{array}$ & $+\frac{7}{33}$ & $+-\frac{7}{33}-$ & $\begin{array}{r}4 \\
+-33-\end{array}$ & $+\frac{4}{33}$ & $+\frac{4}{33}$ & $+-\frac{1}{11}$ & 0 & $-\frac{1}{11}$ & $+\frac{1}{11}$ & 0 & $--\frac{1}{11}$ & 0 \\
\hline 3 & $+\begin{array}{c}1 \\
33\end{array}$ & $\begin{array}{l}7 \\
+\quad 33 \\
-\end{array}$ & $\begin{array}{r}13 \\
+-33 \\
\end{array}$ & $-\frac{2}{33}$ & $+\frac{4}{33}$ & $+\frac{10}{33}$ & $+-{ }^{2}$ & $+\frac{1}{11}$ & 0 & 0 & $-\frac{1}{11}-$ & $-\frac{2}{11}$ & 0 \\
\hline 4 & $+\frac{10}{33}$ & $+\frac{4}{33}$ & $-\frac{2}{33}$ & $+\begin{array}{l}13 \\
33\end{array}$ & $+-\frac{7}{33}-$ & $+\frac{1}{33}-$ & $-\frac{2}{11}$ & $-\frac{1}{11}$ & 0 & 0 & $+\frac{1}{11}$ & $+\frac{2}{11}$ & 0 \\
\hline 5 & $+\frac{4}{33}$ & $+\frac{4}{33}$ & $+\frac{4}{33}$ & $+\frac{7}{33}$ & $+\frac{7}{33}$ & $+\frac{7}{33}$ & $-\frac{1}{11}$ & 0 & $+\frac{1}{11}$ & $\begin{array}{c}1 \\
-11\end{array}$ & 0 & $+-\frac{1}{11}$ & 0 \\
\hline 6 & $-\frac{2}{33}$ & $+\frac{4}{33}$ & $+\frac{19}{33}$ & $+\frac{1}{33}$ & $+\frac{7}{3 s}$ & $+\frac{13}{33}$ & 0 & $+\frac{1}{11}$ & $+\frac{2}{11}$ & $-\frac{2}{11}$ & $-\frac{1}{11}$ & 0 & 0 \\
\hline
\end{tabular}

(II) $\delta Y^{\prime}$

\begin{tabular}{|c|c|c|c|c|c|c|c|c|c|c|c|c|c|}
\hline & $\Delta X_{1}$ & $\Delta X_{2}$ & $\Delta X_{3}$ & $\Delta X_{4}$ & $\Delta X_{5}$ & $\Delta X_{6}$ & $\Delta Y_{1}$ & $\Delta Y_{2}$ & $\Delta Y_{3}$ & $\Delta Y_{4}$ & $\Delta Y_{5}$ & $\Delta Y_{6}$ & $\Delta Z_{1} \sim \Delta Z_{6}$ \\
\hline 1 & 0 & $+\frac{1}{11}$ & $+\frac{2}{11}$ & $-\frac{2}{11}$ & $-\frac{1}{11}$ & 0 & $\begin{array}{r}13 \\
+-33 \\
33\end{array}$ & $+\frac{7}{33}$ & $+\begin{array}{c}1 \\
+33\end{array}$ & $+-\frac{10}{33}$ & $+\frac{4}{33}$ & $\begin{array}{c}2 \\
-33 \\
-3\end{array}$ & 0 \\
\hline 2 & $-\frac{1}{11}$ & 0 & $+\frac{1}{11}-$ & $-{ }_{11}^{1}$ & 0 & $+\cdots 1-$ & $\begin{array}{r}7 \\
+\quad 33\end{array}$ & $\begin{array}{c}7 \\
+33\end{array}$ & $+-\frac{7}{33}-$ & $+\frac{4}{33}-$ & $+\frac{4}{33}$ & $+\frac{4}{33}$ & 0 \\
\hline 3 & $-\frac{2}{11}$ & $-{ }^{-1}-$ & 0 & 0 & $+\frac{1}{11}$ & $+-\frac{2}{11}$ & $+-\frac{1}{33}-$ & $+\frac{7}{33}$ & $\begin{array}{l}13 \\
+-33\end{array}$ & $-\frac{2}{33}$ & $+\frac{4}{33}$ & $+\frac{10}{33}$ & 0 \\
\hline 4 & $+\frac{2}{11}$ & $+\frac{1}{11}$ & 0 & 0 & $-\frac{1}{11}$ & $-\frac{2}{11}$ & $+\frac{10}{33}$ & $+\frac{4}{33}$ & $\begin{array}{c}2 \\
-33\end{array}$ & $+\frac{13}{33}$ & $+\frac{7}{33}$ & $+\frac{1}{33}$ & 0 \\
\hline 5 & $+\frac{1}{11}-$ & 0 & $-\frac{2}{11}$ & $+\frac{1}{11}$ & 0 & $-\frac{1}{11}$ & $+\frac{4}{33}$ & $+\quad \begin{array}{c}4 \\
+33\end{array}$ & $\begin{array}{c}4 \\
+-33\end{array}$ & $+-\frac{7}{33}$ & $+\frac{7}{33}$ & $\begin{array}{r}7 \\
+33\end{array}$ & 0 \\
\hline 6 & 0 & $-\frac{1}{11}$ & $-\frac{1}{11}$ & $+\frac{2}{11}$ & $+\frac{1}{11}$ & 0 & $-\frac{2}{33}$ & $\begin{array}{c}4 \\
+-33\end{array}$ & $+\frac{10}{33}$ & $+\frac{1}{33}$ & $+\frac{7}{33}$ & $+\frac{13}{33}$ & 0 \\
\hline
\end{tabular}

(III) $\delta Z^{\prime}$

\begin{tabular}{|c|c|c|c|c|c|c|c|c|}
\hline & $\Delta X_{1} \sim \Delta X_{6}$ & $\Delta Y_{1} \sim \Delta Y_{6}$ & $\Delta Z_{1}$ & $\Delta Z_{2}$ & $\Delta Z_{3}$ & $\Delta Z_{4}$ & $\Delta Z_{5}$ & $\Delta Z_{6}$ \\
\hline 1 & 0 & 0 & $+\frac{7}{12}$ & $+\frac{1}{3}$ & $+\frac{1}{12}$ & $+\frac{1}{4}$ & 0 & -1 \\
2 & 0 & 0 & $+\frac{1}{3}$ & $+\frac{1}{3}$ & $+-\frac{1}{3}$ & 0 & 0 & 0 \\
3 & 0 & 0 & $+\frac{1}{12}$ & $+\frac{1}{3}$ & $+\frac{7}{12}$ & $-\frac{1}{4}$ & 0 & $+\frac{1}{4}$ \\
4 & 0 & 0 & $+\frac{1}{4}$ & 0 & $-\frac{1}{4}$ & $+\frac{7}{12}$ & $+\frac{1}{3}$ & $+\frac{1}{12}$ \\
5 & 0 & 0 & 0 & 0 & 0 & $+\frac{1}{3}$ & $+\frac{1}{3}$ & $+-\frac{1}{3}$ \\
6 & 0 & 0 & --1 & 0 & $+-\frac{1}{4}$ & $+\frac{1}{12}$ & $+\frac{1}{3}$ & $+\frac{7}{12}$ \\
\hline
\end{tabular}

Table XI 基準点の誤差の影響（30）式を考慮し た場合の座標誤差 Displacement of co-ordinates taking the effect of the error of control points into consideration by Eq. (30)

\begin{tabular}{|l|l|l|}
\hline & (a) $\begin{array}{l}\text { Mono-ocular } \\
\text { comparator }\end{array}$ & (b) $\begin{array}{l}\text { Stereo } \\
\text { comparator }\end{array}$ \\
\hline$\delta X_{1}, 3$ & $\pm 11.2 \mathrm{~cm}$ & $\pm 10.5 \mathrm{~cm}$ \\
$\delta X_{2}$ & \pm 10.5 & \pm 10.2 \\
$\delta X_{4}, 6$ & \pm 11.2 & \pm 13.1 \\
$\delta X_{5}$ & \pm 10.5 & \pm 13.6 \\
\hline$\delta Y_{1}, 3$ & \pm 14.8 & \pm 12.9 \\
$\delta Y_{2}$ & \pm 12.0 & \pm 13.7 \\
$\delta Y_{4}, 6$ & \pm 14.8 & \pm 13.3 \\
$\delta Y_{5}$ & \pm 12.0 & \pm 11.5 \\
\hline$\delta Z_{1},{ }_{3},{ }_{4}, 6$ & \pm 26.3 & \pm 21.4 \\
$\delta Z_{2},{ }_{5}$ & \pm 24.4 & \pm 19.0 \\
\hline
\end{tabular}

Table XII 基準点の誤差の影響（31）式を考虑 した場合の基準点の閉塞 Closure of control points taking the effect of the error of control points into consideration by Eq. (31)

\begin{tabular}{|c|c|c|}
\hline & $\begin{array}{l}\text { (a) Mono-ocular } \\
\text { comparator }\end{array}$ & $\begin{array}{l}\text { (b) Stereo- } \\
\text { comparator }\end{array}$ \\
\hline$\partial X_{1}, 3-\Delta X_{1},_{3}$ & $\pm 12.5 \mathrm{~cm}$ & $\pm 11.9 \mathrm{~cm}$ \\
\hline$\delta X_{2}-\Delta X_{2}$ & \pm 12.9 & \pm 12.7 \\
\hline$\delta X_{4},{ }_{6}-\Delta X_{4},{ }_{6}$ & \pm 12.5 & \pm 14.2 \\
\hline$\partial X_{5}-\Delta X_{5}$ & \pm 12.9 & \pm 15.6 \\
\hline$\grave{o} Y_{1 \cdot 3}-\Delta Y_{1}, 3$ & \pm 15.9 & \pm 14.1 \\
\hline$\delta Y_{2}-d Y_{2}$ & \pm 14.2 & \pm 15.7 \\
\hline$\delta Y_{4},{ }_{6}-\Delta Y_{4},{ }_{6}$ & \pm 15.9 & \pm 14.2 \\
\hline$\delta Y_{5}-\Delta Y_{5}$ & \pm 14.2 & \pm 13.8 \\
\hline$\delta Z_{1},{ }_{3},{ }_{4},{ }_{6}-\Delta Z_{1},{ }_{3},{ }_{4}, 6$ & \pm 24.4 & \pm 20.5 \\
\hline$\delta Z_{2},-\Delta Z_{2}, 5$ & \pm 27.1 & \pm 22.4 \\
\hline
\end{tabular}


の偏位より，基準点への閉塞誤差の方が外見上大きく 現われるととが分る。換言すれば，基準点の閉塞差が 大きい場合には，必ずしも対応する点のモデル座標算 出のための写真測定の誤差が大きいためのみでなく， 基準点の测地座標そのものの誤差が大きい場合が含ま れることに注意しなければならない。いま

$$
\nu=10 \mathrm{~cm}, \rho=20 \mathrm{~cm}
$$

とすると

$$
\begin{aligned}
& \delta X^{\prime}{ }_{1,3,4}, 6, \quad \delta Y^{\prime}{ }_{1,3,4},{ }_{6}= \pm 5.4 \mathrm{~cm} \\
& \delta Z^{\prime}{ }_{1,3,4},{ }_{6}= \pm 14.4 \mathrm{~cm} \\
& \delta X^{\prime}{ }_{2,5}, \quad \delta Y^{\prime}{ }_{2,5}= \pm 4.6 \mathrm{~cm} \\
& \delta Z^{\prime}{ }_{2,5}= \pm 11.4 \mathrm{~cm}
\end{aligned}
$$

および

$$
\begin{aligned}
& \left.\begin{array}{l}
\delta X^{\prime}{ }_{1,3,4,6}-\Delta X_{1,3,4,6} \\
\delta Y^{\prime}{ }_{1,3,4,6}-\Delta Y_{1,3,4,6}
\end{array}\right\}= \pm 7.8 \mathrm{~cm}, \\
& \delta Z^{\prime}{ }_{1,3,4,6}-\Delta Z_{1},{ }^{3}{ }_{4,4}= \pm 13.0 \mathrm{~cm} \\
& \left.\begin{array}{l}
\delta X^{\prime}{ }_{2,5}-\Delta X_{2,5} \\
\delta Y^{\prime}{ }_{2,5}-\Delta Y_{2,5}
\end{array}\right\}= \pm 8.9 \mathrm{~cm}, \\
& \delta Z^{\prime}{ }_{2,5}-\Delta Z_{2,5}= \pm 16.4 \mathrm{~cm}
\end{aligned}
$$

となる。

座標䛊差は(21)によって表わされるので，今までに えられた座標䛊差を与える諸表 (Tables III あるいは VI および X) を考虑しつつ議論をすすめていかなけ ればならない。今与点の誤差による影響が(30)式で与

\begin{tabular}{|c|c|c|c|}
\hline & & \multicolumn{2}{|c|}{$\Delta$} \\
\hline & & $\begin{array}{l}\text { (a) Mono-ocular } \\
\text { comparator }\end{array}$ & $\begin{array}{l}\text { (b) Stereo- } \\
\text { comparator }\end{array}$ \\
\hline$\delta X_{1}, 3 \quad k$ & に対して & $4.4 \mathrm{~cm}$ & $4.0 \mathrm{~cm}$ \\
\hline$\delta X_{2}$ & " & 4.2 & 4.1 \\
\hline$\delta X_{4},{ }_{6}$ & " & 4.4 & 5.4 \\
\hline$\delta X_{5}$ & " & 4.2 & 5.8 \\
\hline$\delta Y_{1}, 3$ & " & 6.2 & 5.3 \\
\hline$\delta Y_{2}$ & $"$ & 5.0 & 5.8 \\
\hline$\delta Y_{4},{ }_{6}$ & $" \prime$ & 6.2 & 5.4 \\
\hline$\delta Y_{5}$ & $" \prime$ & 5.0 & 4.7 \\
\hline$\delta Z_{1},{ }_{3},{ }_{4},{ }_{6}$ & " & 11.5 & 6.9 \\
\hline$\delta Z_{2},{ }_{5}$ & " & 10.8 & 6.8 \\
\hline
\end{tabular}
えられている場合, 相互標定の誤差による影響が

Table V あるいはVIII の最右端によって与えられて いるものとすると，乙れらを統計的に相加えることに よって Table XI の如き值をうる。また(22)によって 基準点の閉塞は Table XII となる。

Table XIII 与点誤差の影影 $\Delta$ The effect of the error of control points

$\left(\varepsilon, \varepsilon^{\prime}=10 \mu\right.$, Base $\left.=800 \mathrm{~m}, \mathrm{a}=0.1\right)$

結び 本報告では, 写真座標あるいは視差の測定值 の誤差が写真の標定におよぼす影響を，極めて簡単な モデルを絶対標定した場合の基準点の閉塞の状況によ

\section{3. 与点誤差の制限}

前項では测地座標の誤差の絶対標定におよぼす影響 について述べたが，逆に最終所要精度を基にして写真 の測定精度およびこれを有効に用いるために与点とし て用いるべき測地座標の誤差に制限を与える目安を立 てるととができる。

写真の標定誤差による座標の一成分の誤差を 七 $\delta$, 与点の誤差による同じ成分の座標誤差を $\pm \Delta$ とする と，とれらによる全体の誤差は

$$
\pm \sqrt{\delta^{2}+\Delta^{2}}
$$

である。 $\Delta$ による影響の因子を $a$ とし

$$
\sqrt{\delta^{2}+\Delta^{2}}=(1+a) \delta
$$

とおいたとき， $a$ による影響ができるだけ少いととが 望ましい。(32)より

$$
\Delta=\delta \sqrt{2 a+a^{2}}
$$

をうる。ここで $a^{2}$ の項を省略すれば

$$
\Delta \doteqdot \sqrt{2 a} \cdot \delta
$$

となる。ઠを定めるとととにより例えば Table V あ るいはVIII によって写真測定に要求される䛊差の範 囲 $\varepsilon$ あるいは $\varepsilon^{\prime}$ を定めることができる。一方，(33） あるいは(34)式によって よってレおよび $\rho$ を定めることができる。これによっ て用いるべき基準点の種類が決定される。

$$
a=0.1 \text { とすると } \Delta=0.45 \delta
$$

となり, 基線長 $800 \mathrm{~m}$ の場合に $\varepsilon=10 \mu, \quad \varepsilon^{\prime}=10 \mu$ とすると(35)を Tahle V あるいはVIII に適用して の大いさが Table XIII の如くに求められる。従って

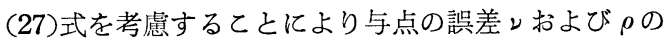
大いさが Table XIV の如くに制限される。

Table XIV 与点誤差の制限 Limitation of the error of control points $\left(\varepsilon, \varepsilon^{\prime}=10 \mu\right.$, Base $\left.=800 \mathrm{~m}, \mathrm{a}=0.1\right)$

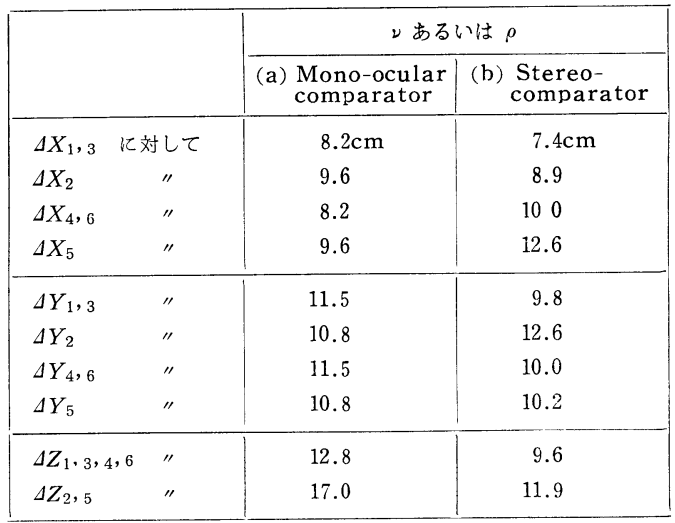

って論じた。

同じ方法を応用して，与点となる測地座標の俁差が 標定におよぼす影響を計算した。また，逆に標定誤差 
Table XV 与点誤差を考虑しない場合座標比較器法とステレオコンパレーター法による测定の 際の諸誤差の比較 Comparison of errors expected in two methods (monoocular co-ordinate comparator method and stereo-comparator method) reduced from Tables $\mathrm{V}$ and VIII, where the error of geodetic co-ordinates of control points is disregarded. The table shows the ratio of the former versas the latter.

\begin{tabular}{|l|l|l|l|l|l|}
\hline$\delta X_{1}, 3$ & $1.08 \times \varepsilon / \varepsilon^{\prime}$ & $\delta Y_{1}, 3$ & $1.18 \times \varepsilon / \varepsilon^{\prime}$ & $\delta Z_{1},{ }_{3}, 4,6$ & $1.30 \times \varepsilon / \varepsilon^{\prime}$ \\
$\delta X_{2}$ & 1.02 & $\delta Y_{2}$ & 0.86 & $\delta Z_{2}, 5$ & 1.41 \\
$\delta X_{4}, 6$ & 0.82 & $\delta Y_{4}, 6$ & 1.16 & & \\
$\delta X_{5}$ & 0.74 & $\delta Y_{5}$ & 1.05 & & \\
\hline
\end{tabular}

を推定するととによって，与点の誤差に限界を与える こと，いいかえれば与点として用いる測地基準点の種 類を選定する基準を与えるととができるととを例によ って述べた。第而節において，その第 1 項の観測方法 (mono-ocular co-ordinate comparator) と第 2 項 の場合（stereo-comparator）とを比較するために, Table V と VIII に扔いて, 一様な観測誤差のある場 合に座標誤差の大きさの比をとると Table XV の如 くになる。同表より見られることは，両者において $\varepsilon=\varepsilon^{\prime}$ と仮定すれば, $\delta X$ および $\delta Y$ につては, 両 者の特失は相半ばするが, $\delta Z$ については, 後者の方 法による場合の方が極めて小さくなるてとである。以 上のととは与点誤差による影響を考慮しない場合であ るが，乙れを考慮した場合についてす，(34)式の制限 に基ずいて与点誤差が与えられている場合には, 両者 の比は Tahle XV と同じになることは当然であり, (29)式で与点䛊差が与えられている場合には, Table XI の最右列に与えられている如き值となる。
本報告ではふれなかったのであるが，測地座標を求 めようとする点（新点, あるいは空中図根点）の䛠差 を, 写真座標あるいは視差の測定值および与点誤差の 関数として求めることは，(10)式に新点の $x, y, z$ を 代入すればよく，乙れについては何ら特別なる問題は ないととを付記する。

\section{参 考 文 献}

[1] 村岡一男 : 解析写真測量に打ける相互標定に ついて (II) 誤差 (1), 写真測量, 第 3 号 (1962), pp. 54-62。

[2]村岡一男: 解析写真量における相互標定につ いて(III)誤差 (2), 写真測量, 第 4 号(1962) pp. $31-40$ 。

［3] 村岡一男: 接続標定に㧍ける誤差について (I) 写真測量, 第 4 号 (1963), pp. 7-17。

[4] 村岡一男: 接続標定に扮ける誤差について （II)写真測量, 第 1 号 (1964), pp. 11-20。 